Vila-Maldonado, S.; Sáez-Gallego, N.M.; García-López, L.M.; Contreras, O.R. (2019) Visual Behavior Influence on Decision in Volleyball Blocking. Revista Internacional de Medicina y Ciencias de la $\begin{array}{lllllll}\text { Actividad Física y el Deporte vol. } 19 & \text { (75) pp. } & \text { 489-504 }\end{array}$ Http://cdeporte.rediris.es/revista/revista75/artinfluencia1060.htm

DOI: $10.15366 /$ rimcafd2019.75.007

\title{
ORIGINAL
}

\section{INFLUENCIA DEL COMPORTAMIENTO VISUAL EN LAS DECISIONES EN EL BLOQUEO DE VOLEIBOL}

\section{VISUAL BEHAVIOR INFLUENCE ON DECISION IN VOLLEYBALL BLOCKING}

\author{
Vila-Maldonado, S. ${ }^{1}$; Sáez-Gallego, N.M.2; García-López, L.M. ${ }^{3}$ y Contreras, \\ O.R. ${ }^{4}$ \\ 1 Doctora en Ciencias de la Actividad Física y del Deporte. Facultad de Ciencias del Deporte. \\ Universidad de Castilla-La Mancha (España) Sara.Vila@uclm.es \\ 2 Doctora en Ciencias de la Actividad Física y del Deporte. Facultad de Educación de Toledo. \\ Universidad de Castilla-La Mancha (España) Nieves.Saez@uclm.es \\ 3 Doctor en Ciencias de la Actividad Física y del Deporte. Facultad de Educación de Albacete. \\ Universidad de Castilla-La Mancha (España) LuisMiguel.Garcia@uclm.es \\ 4 Doctor en Derecho. Facultad de Educación de Albacete. Universidad de Castilla-La Mancha \\ (España) Onofre.CJordan@uclm.es
}

Código UNESCO: 6199 Otras especialidades psicológicas (Psicología del deporte). Clasificación del Consejo De Europa: 15. Psicología del Deporte

Recibido 9 de noviembre de 2017 Received November 9, 2017

Aceptado 26 de marzo de 2018 Accepted March 26, 2018

\section{RESUMEN}

El objetivo del presente estudio fue conocer la influencia del comportamiento visual (localización, número y duración de las fijaciones visuales) sobre la toma de decisiones en la acción del bloqueo en voleibol. Se analizó el comportamiento visual de 38 jugadoras de voleibol (23.9 \pm 4.2 años), que competían de manera federada, mediante el sistema de registro de movimientos oculares (Mobile Eye) durante la visualización de secuencias de video proyectadas a tamaño real. Los modelos de regresión obtenidos muestran que se obtienen mayores porcentajes de acierto cuando la duración de las fijaciones es reducida en las zonas $\mathrm{HC}$ y CB, y cuando aumenta el número de fijaciones en la zona BM y disminuye en la zona CB. 
PALABRAS CLAVE: toma de decisiones, comportamiento visual, bloqueo, voleibol.

\section{ABSTRACT}

The purpose of this study was to determine the influence of visual behaviour (visual fixation location, number and duration) on decision-making in volleyball blocking. The visual behavior of 38 volleyball players ( $23.9 \pm 4.2$ years), who took part in federated competition, was analyzed with the eye tracking system (Mobile Eye) during visualization of real size video sequences. Regression models show that higher success percentages were obtained when the duration of fixations is low in $\mathrm{HC}$ and $\mathrm{CB}$ areas, and when the number of fixations increases in BM zone and decreases in CB zone.

KEY WORDS: decision making, visual behavior, blocking, volleyball.

\section{INTRODUCCIÓN}

El canal visual es el más utilizado en el procesamiento de información de habilidades de carácter abierto, y por tanto el canal dominante en el proceso de optimización del rendimiento deportivo a través de la utilización de la información (Damas, Moreno, Reina y Del Campo, 2004; Del Campo, Reina, Sanz, Fuentes y Moreno, 2003; Moreno, Ávila y Damas, 2001; Ruiz y Sánchez, 1997). La relación entre percepción visual y toma de decisiones es evidente, los procesos de atención están determinados por las estrategias visuales usadas para analizar el entorno y tomar la información para su procesamiento (Tenebaum, 2003). Esta relación aumenta en un entorno variable en el que los móviles adquieren velocidades elevadas y trayectorias complejas. En estos deportes, el déficit de tiempo hace que la velocidad con la que se realiza la toma de decisiones sea crucial para el éxito de la acción, proceso para el cuál una adecuada percepción visual es imprescindible (Abernethy, 1988).

El proceso de búsqueda visual se caracteriza por una detección inicial del objeto dentro de la visión periférica, que proporciona al deportista una información acerca de "dónde está". Esta información es procesada rápidamente para facilitar la detección del movimiento y otros eventos del entorno visual (Knudson y Kluka, 1997). Esa detección del estímulo en la periferia se asume que no es realizada conscientemente, sin embargo la visión periférica también puede usarse de forma consciente durante el proceso de búsqueda visual (Palmi, 2007). Tras la detección del estímulo, las áreas de mayor significación informativa son llevadas a la región fóvea (Quevedo y Solé, 2007), dónde se producen las fijaciones visuales, y la duración de éstas parece señalar la importancia relativa que tiene esa área de la 
imagen para el deportista. Una fijación ocurre cuando la mirada está centrada sobre un objeto o localización dentro de su ángulo visual, durante al menos $100 \mathrm{~ms}$ (Vickers, 2007). Los investigadores sugieren que durante las fijaciones visuales, se lleva a cabo el procesamiento de la información procedente del entorno, de modo que cuanto mayor sea la duración de la fijación visual, mayor será la información procesada (Just y Carpenter, 1976). La duración y número de las fijaciones cambia en función del tipo de tarea que se desempeñe o del entorno que se perciba, de este modo si la tarea observada aumenta en dificultad, también lo hará el número de fijaciones visuales. Entre fijaciones se producen los movimientos sacádicos, que son los movimientos rápidos del ojo para redirigirse de un punto a otro del campo visual, llevando un nuevo objeto del entorno a la región central de la retina.

Por tanto, conocer la fijación de la mirada es importante ya que lo que está siendo fijado por los ojos indica aquello que está siendo procesado por la mente (Scheiter y Van Gog, 2009). Para poder actuar con efectividad en entornos deportivos con incertidumbre, los jugadores deben focalizar su visión solamente hacia aquellas zonas más relevantes del juego (Moreno, Del Campo, Reina, Ávila, y Sabido, 2003).

Las investigaciones revisadas acerca de la estrategias visuales de los deportistas, apuntan a que los expertos usan menos fijaciones de mayor duración, incluyendo periodos prolongados de "tranquilidad" visual (Mann, Williams, Ward y Janelle, 2007). También son capaces de interpretar de forma más eficiente la información visual disponible, en el área que dominan. Estas diferencias se encuentran en ambientes y acciones relacionadas con el deporte en concreto, y no se dan cuando las capacidades visuales se estudian de forma general (Abernethy, Neal y Koning, 1994; Kioumourtzoglou, Derri, Tzetzis y Therodorakis, 1998; Kioumourtzoglou, Michalopoulou, Tzetzis y Kourtessis, 2000). Así podríamos concluir que una estrategia visual efectiva es aquella con un menor número de fijaciones visuales, de mayor duración, y pocos movimientos sacádicos (Bard y Fleury, 1976; Vickers, 1988; Abernethy, 1990; Savelsbergh, Williams, Van der Kamp y Ward, 2002; Williams, Singer y Frehlich, 2002; Vaeyens, Lenoir, Williams, Mazyn y Philippaerts, 2007; Vaeyens, Lenoir, Williams y Philippaerts, 2007; Reina, Moreno y Sanz, 2007; Mann et al., 2007), ya que los jugadores dispondrían así de mayor tiempo de procesamiento de la información. Sin embargo, el ratio de búsqueda visual puede variar en función de aspectos como la modalidad deportiva (Williams, Davids, Burwitz, y Williams, 1993; Williams y Davids, 1995), el rol desempeñado (ataque o defensa) (Williams, 2000; Vaeyens, Lenoir, Williams, Mazyn et al, 2007; Afonso, Garganta, McRobert, Williams y Mesquita, 2012), la cantidad de incertidumbre de la escena (Bard y Fleury, 1976) o la proximidad al objetivo (Roca, Ford, McRobert y Williams, 2013).

Con todo ello, si queremos avanzar en un deporte en concreto, resulta necesario realizar investigaciones se lleven a cabo de forma específica, en modalidades y situaciones precisas. Según Hayhoe (2004), el tipo de fijaciones visuales y su 
duración depende de factores variados, entre ellos el tipo de tarea y el tiempo del que disponemos para captar la información relevante. Por este motivo los resultados obtenidos en una acción puntual, no pueden generalizarse para todas las acciones deportivas. Por tanto dentro de cada acción se debería extraer una estrategia de búsqueda visual, al conocer cómo los expertos usan sus movimientos oculares para extraer información visual importante, en acciones deportivas concretas. Así estas estrategias podrían ser usadas para entrenar el comportamiento visual de los deportistas con menos experiencia (Liebermann et al., 2002).

En el caso del voleibol, autores como Sellinger y Ackerman (1985) afirman que la relación entre el balón y el colocador, permite al bloqueador conocer la intención de éste jugador, en cuanto a la zona a la que enviará el pase y el tiempo de colocación, y de esta forma poder anticiparse y llegar al lugar concreto a tiempo para contrarrestar la acción de ataque. Por tanto, si hablamos de la acción del bloqueo, resulta de especial importancia centrarnos en la figura del colocador, como fuente principal de aporte informativo.

La presente investigación se centra en conocer la información visual en la que centra su atención la bloqueadora, ampliando de este modo el trabajo realizado por Vila-Maldonado, Sáez-Gallego, Abellán y García-López (2014) en el que se analizaba la toma de decisiones en dicha acción, para conocer así la relación entre el éxito en sus decisiones y su comportamiento visual. El objetivo del trabajo, por tanto, es conocer si el número y tiempo de fijaciones visuales de las participantes influye de alguna manera en el acierto o error en su toma de decisiones. De este modo pretendemos aportar a los entrenadores datos que resulten de utilidad para formar a las jóvenes promesas de este deporte, y mejorar el rendimiento de los jugadores y jugadoras de voleibol en general.

\section{MATERIAL Y MÉTODOS}

\section{PARTICIPANTES}

Se utilizó una muestra intencional compuesta por 38 jugadoras de voleibol $(23.9 \pm 4.2$ años) que competían de manera federada. Todas las jugadoras que realizaron el estudio participaban en la acción de bloqueo. 29 de ellas eran jugadoras de diversos clubes militantes en primera y segunda división y las 9 restantes procedían de la selección española absoluta.

El estudio fue aprobado por la Real Federación Española de Voleibol, el seleccionador nacional, y los entrenadores de los clubes participantes.

Para la realización de las secuencias de colocación se precisó la colaboración de colocadoras experimentadas con el mismo nivel de juego que las participantes del 
estudio. De este modo participaron dos colocadoras pertenecientes a la Selección Española Absoluta (10 y 17 veces internacionales, cada una de ellas) y dos colocadoras de primera división (8 y 12 años de experiencia deportiva en voleibol).

\section{VARIABLES DEL ESTUDIO}

\section{VARIABLE INDEPENDIENTE}

La variable independiente es el éxito en la toma de decisiones. Dicha variable procede de los resultados hallados en el trabajo previo realizado por Vila-Maldonado et al. (2014) y se entiende como la relación de respuestas correctas que consiguen las jugadoras una vez que predicen y deciden hacia qué zona enviará el balón la colocadora.

Esta variable se divide en porcentaje de aciertos en zona 3 (\%A3), porcentaje de aciertos en zona $4(\% \mathrm{~A} 4)$ y porcentaje de aciertos totales (\%AT).

\section{VARIABLES DEPENDIENTES}

La variable dependiente fue el comportamiento visual, y dentro de él se han analizado las fijaciones visuales (Vickers, 2007). También hemos entendido como fijaciones los movimientos de seguimiento, que son aquellos que mantienen una imagen estable de un objeto en la retina mientras se realiza un movimiento ocular. Dentro de las fijaciones visuales, se ha analizado su localización, el número de fijaciones y su duración en segundos. En relación a la localización, se han tenido en cuenta 8 áreas ampliadas de la escena y de la colocadora que se especifican en la figura 1, así como las fijaciones localizadas en el fondo de la escena o en otras zonas sin relevancia informativa. Se ha analizado la media y el porcentaje del número y la duración de las fijaciones por participante y por localización concreta 


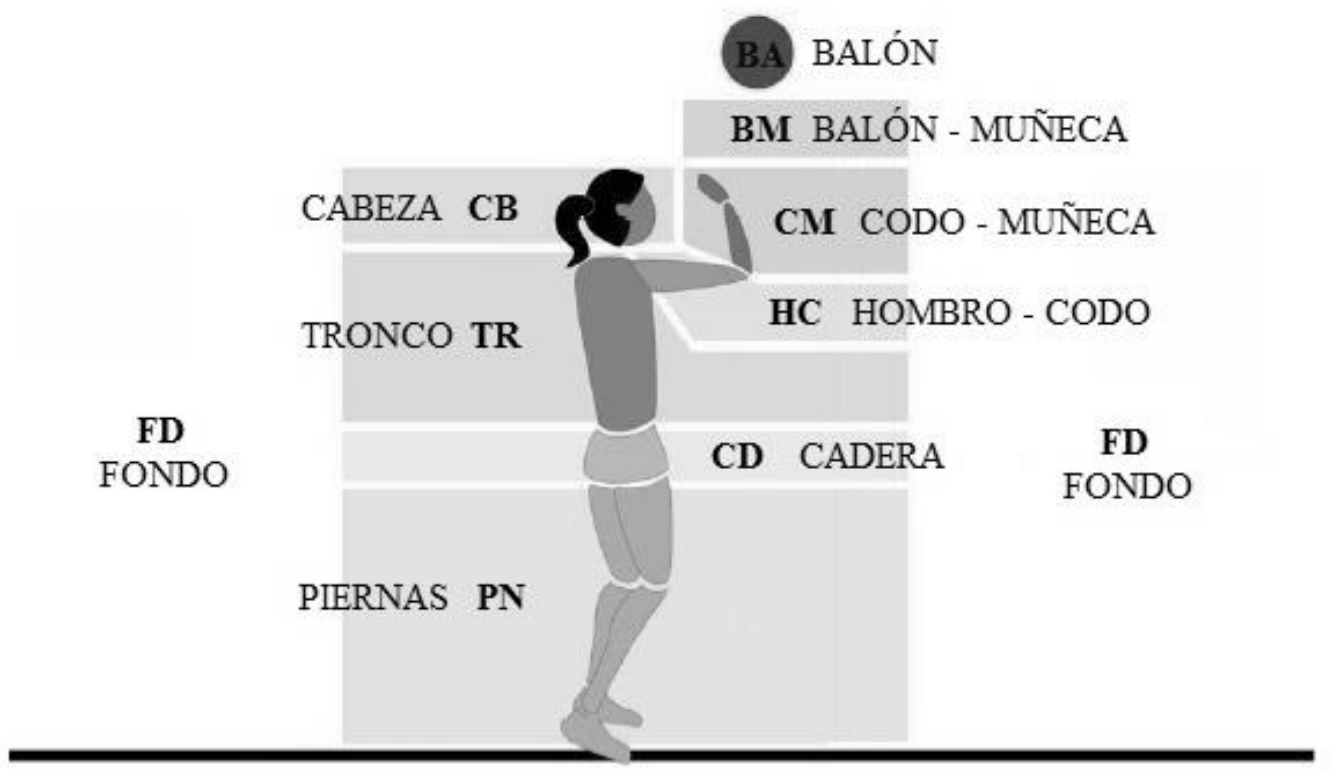

OT OTROS (Otras zonas diferentes a las ya descritas)

Figura 1. Representación gráfica de las distintas localizaciones.

\section{INSTRUMENTOS}

El estudio se desarrolló en la pista de voleibol de los diferentes pabellones polideportivos de entrenamiento de los equipos. En ella se ubicó el siguiente material (ver figura 2): postes y red reglamentarios, a medio metro de la red se situó una pantalla de retroproyección de $5 \times 3 \mathrm{~m}$, y por detrás de ella un cañón proyector DLP modelo BENQ PB2250 de 2200 ANSI lumens - XGA (1024 x 768), también se utilizó un ordenador portátil HP Compaq 6710b, para la proyección de escenas, y con el fin de grabar el experimento utilizamos una videocámara digital con formato minidv, modelo SONY DCR - TRV15E PAL, y un trípode modelo HAMA STAR 62. 


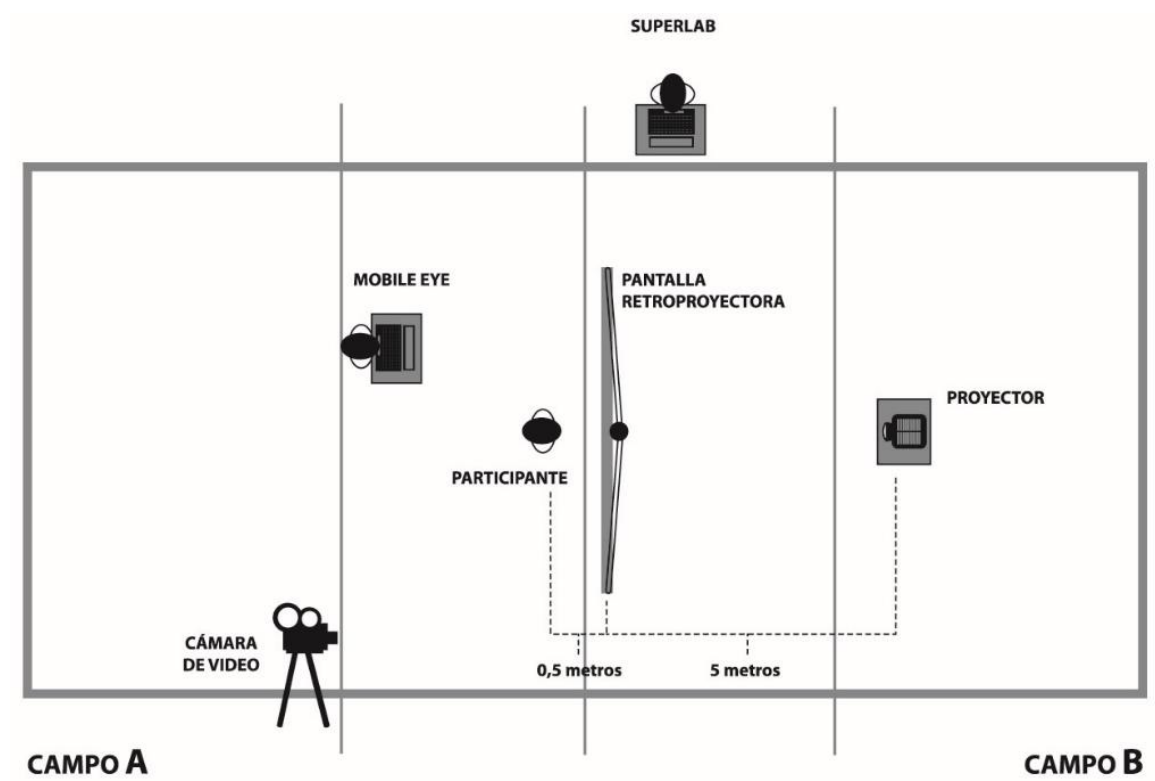

Figura 2. Esquema de la disposición del material.

Con el objeto de medir el comportamiento visual de las participantes se utilizó un Sistema de Seguimiento de Movimientos Oculares Mobile Eye de los laboratorios ASL (Bedford, USA).

Las secuencias de colocación que se proyectaron a las jugadoras se editaron con el software Pinnacle Studio Plus 9.3, y se presentaron con el programa de presentación de estímulos Superlab. 4.0. Los videos resultantes de sistema Mobile Eye se analizaron fotograma a fotograma con el software VirtualDub-1.9.6.

\section{PROCEDIMIENTO}

\section{SECUENCIAS DE VIDEO}

4 colocadoras, del mismo nivel competitivo que las participantes, participaron en la grabación de las secuencias. Se les pidió que realizasen colocaciones de segundo tiempo a zona 3 y de tercer tiempo a zona 4 , tal y como lo harían en una situación de juego. Se editaron un total de 48 secuencias de colocación, 12 de ellas para los ensayos de prueba y 36 para los de medida. Las secuencias se cortaron al finalizar la primera parte del vuelo del balón, tras la fase post-contacto (ver Vila-Maldonado et al., 2014).

\section{SITUACIÓN EXPERIMENTAL}

La participante se situaba en la zona 3, a 0.5 metros de la red, y en posición de espera para la acción de bloqueo (figura 2), con el sistema de seguimiento de 
movimientos oculares colocado. Al inicio del experimento, todas las jugadoras recibieron las mismas instrucciones acerca del procedimiento a través de una proyección en la pantalla, y se llevó a cabo la calibración del Mobile Eye. Ésta se repitió al final del experimento para comprobar que no se había visto afectada durante la toma de datos.

Tras la calibración se proyectaban las secuencias de prueba y medida en las que la participante debía observar lo que se mostraba en la pantalla y, en función de ello, tomar la decisión acerca del lugar donde estimaba que iría dirigida la colocación.

\section{ANÁLISIS DE LOS DATOS}

Se eliminaron dos secuencias de colocación por presentar errores que no nos permitían su análisis en al menos el $80 \%$ de la muestra, por lo que el número final de secuencias de medida fue de 34 .

El análisis de los videos del Mobile Eye fue realizado por dos investigadoras de forma independiente, aplicando el protocolo correspondiente para consensuar las posibles diferencias en la extracción de los datos. Se utilizó para ello una planilla de observación de Excel.

Los datos resultantes se analizaron en SPSS Versión 19.0. Se analizaron parámetros descriptivos del número de fijaciones visuales y de los tiempos de duración (medias y desviaciones típicas). Se realizaron modelos de regresión manteniendo todas las variables para que el proceso de tamizado las rechazarse o por el contrario las mantuviese en el modelo. Se ha utilizado como criterio práctico para que una variable pueda mantenerse dentro del modelo, el resultado de la prueba t (prueba de calidad de coeficientes) para $p \leq 0,05$. Dentro de los modelos de regresión se ha efectuado la prueba $\mathrm{F}$ de Fisher.

\section{RESULTADOS}

En la tabla 1 se muestran los parámetros descriptivos $(\mathrm{M} \pm \mathrm{DT})$ del número y la duración total de las fijaciones, en función de su localización. 
Rev.int.med.cienc.act.fís.deporte - vol. 19 - número 75 - ISSN: 1577-0354

Tabla 1. Estadísticos descriptivos ( $\mathrm{M} \pm \mathrm{DT}$ ) del número y duración de las fijaciones por zona.

\begin{tabular}{ccc}
\hline Localizaciones & $\begin{array}{c}\text { Número de fijaciones } \\
(\mathrm{M} \pm \mathrm{DT})\end{array}$ & $\begin{array}{c}\text { Duración de las } \\
\text { fijaciones }(\mathbf{s}) \\
(\mathrm{M} \pm \mathrm{DT})\end{array}$ \\
\hline BALÓN (BA) & $0,33 \pm 0,3$ & $0,349 \pm 0,183$ \\
BALÓN-MUÑ̃CA (BM) & $0,19 \pm 0,2$ & $0,366 \pm 0,258$ \\
CODO-MUÑECA (CM) & $0,60 \pm 0,3$ & $0,491 \pm 0,103$ \\
HOMBRO-CODO (HC) & $0,39 \pm 0,4$ & $0,549 \pm 0,328$ \\
CABEZA (CB) & $0,46 \pm 0,3$ & $0,545 \pm 0,247$ \\
TRONCO (TR) & $0,08 \pm 0,1$ & $0,404 \pm 0,355$ \\
CADERA (CD) & $0,03 \pm 0,1$ & $0,147 \pm 0,240$ \\
PIERNAS (PN) & $0,07 \pm 0,2$ & $0,194 \pm 0,299$ \\
FONDO (FD) & $1,01 \pm 0,1$ & $0,382 \pm 0,125$ \\
OTROS (OT) & $0,10 \pm 0,3$ & $0,261 \pm 0,296$ \\
TOTAL & 3,3 & 3,689 \\
\hline
\end{tabular}

En la figura 3 se muestra la distribución por localizaciones del total de las fijaciones realizadas.

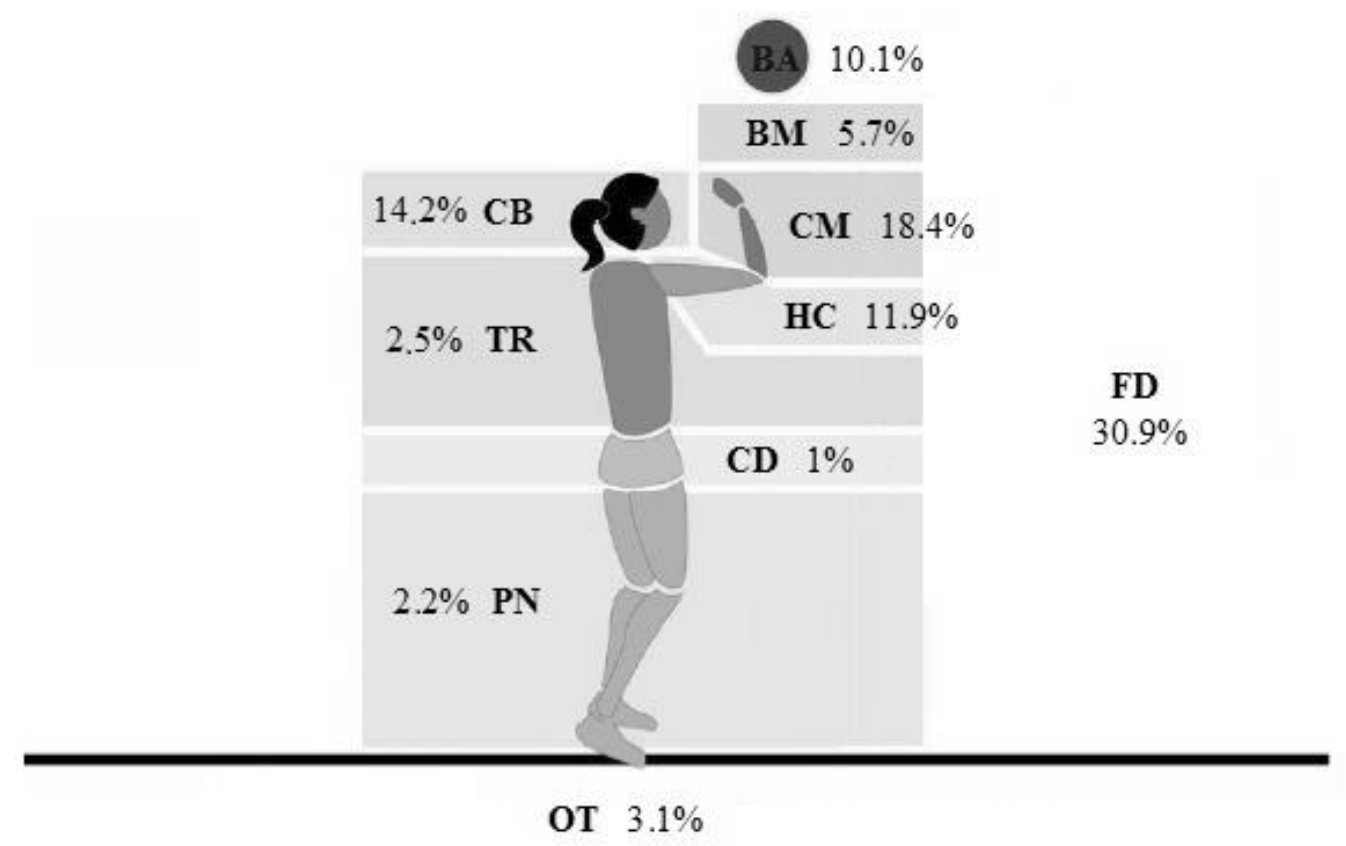

Figura 3. Porcentajes del total de fijaciones en cada zona.

Para conocer cómo influye sobre el porcentaje de aciertos (\%A3, \%A4 y \%AT) la media del número y duración de las fijaciones en las distintas zonas de visualización, se han realizado modelos de regresión. 
En cuanto a la duración media de las fijaciones, para el porcentaje de aciertos totales se ha obtenido el modelo de regresión múltiple que se muestra en la tabla 2. Los resultados indican que los tiempos de duración de las fijaciones en zonas $\mathrm{HC}$ y CB afectan negativamente al éxito total en la toma de decisiones en esta acción. Según el modelo obtenido, los mejores porcentajes de aciertos totales pueden obtenerse empleando el menor tiempo posible de fijación en estas zonas.

Tabla 2. Modelo de regresión en función de la duración. Variable dependiente: \%AT.

\begin{tabular}{|c|c|c|c|c|c|c|}
\hline & & \multicolumn{2}{|c|}{$\begin{array}{l}\text { Coeficientes no } \\
\text { estandarizados }\end{array}$} & \multirow{2}{*}{$\begin{array}{c}\begin{array}{c}\text { Coeficientes } \\
\text { estandarizados }\end{array} \\
\text { Beta }\end{array}$} & \multirow[t]{2}{*}{$T$} & \multirow[t]{2}{*}{$p$} \\
\hline & & $B$ & E.T. & & & \\
\hline & $\begin{array}{l}\text { Duración (s) } \\
\text { HOMBRO-CODO (HC) }\end{array}$ & -41.897 & 8.429 & $-0,896$ & -4.971 & 0,000 \\
\hline & $\begin{array}{l}\text { Duración (s) } \\
\text { CABEZA (CB) }\end{array}$ & -19.815 & 7.794 & $-0,319$ & -2.542 & 0,016 \\
\hline $\begin{array}{l}R^{2}: \\
0,528\end{array}$ & & & & & & \\
\hline
\end{tabular}

Del mismo modo sucede con los aciertos en zona 3 (tabla 3 ) y con los aciertos en zona 4 (tabla 4), donde se confirma que los mejores porcentajes de aciertos se obtienen si se emplea el menor tiempo posible en las zonas $\mathrm{HC}$ y CB.

Tabla 3. Modelo de regresión en función de la duración. Variable dependiente: \%A3.

\begin{tabular}{|c|c|c|c|c|c|}
\hline & \multicolumn{2}{|c|}{$\begin{array}{l}\text { Coeficientes no } \\
\text { estandarizados }\end{array}$} & \multirow{2}{*}{$\begin{array}{c}\begin{array}{c}\text { Coeficientes } \\
\text { estandarizados }\end{array} \\
\text { Beta } \\
\end{array}$} & \multirow[t]{2}{*}{$\mathbf{T}$} & \multirow[t]{2}{*}{$p$} \\
\hline & $B$ & E.T. & & & \\
\hline $\begin{array}{l}\text { Duración (s) } \\
\text { HOMBRO-CODO (HC) }\end{array}$ & -24.802 & 7.979 & $-0,44$ & -3.109 & 0,004 \\
\hline $\begin{array}{l}\text { Duración (s) } \\
\text { CABEZA (CB) }\end{array}$ & -21.756 & 10.613 & $-0,29$ & -2.05 & 0,048 \\
\hline
\end{tabular}


Rev.int.med.cienc.act.fís.deporte - vol. 19 - número 75 - ISSN: 1577-0354

Tabla 4. Modelo de regresión en función de la duración. Variable dependiente: \%A4.

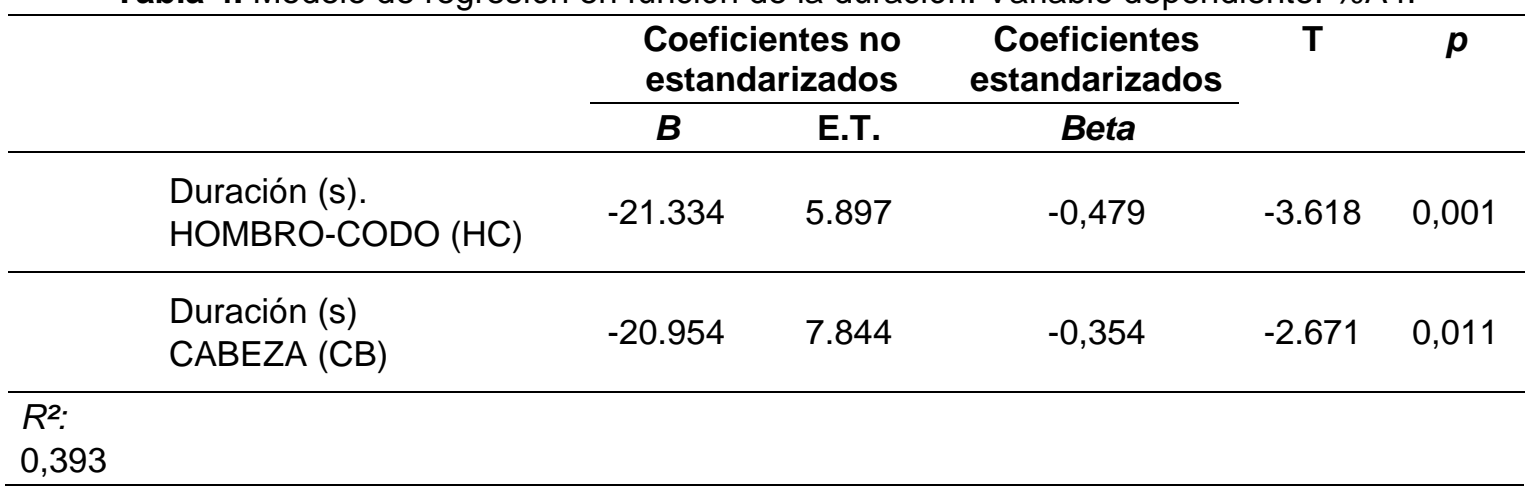

En relación al número medio de fijaciones, el \%AT se ve influenciado de forma positiva por el número medio de fijaciones en zona BM y de forma negativa por el número medio de fijaciones en zona CB (tabla 5).

Tabla 5. Modelos de regresión en función del número de fijaciones. Variable dependiente: \%AT.

\begin{tabular}{|c|c|c|c|c|c|c|}
\hline & & $\begin{array}{l}\text { Coefic } \\
\text { estan }\end{array}$ & $\begin{array}{l}\text { ntes no } \\
\text { rizados }\end{array}$ & $\begin{array}{c}\text { Coeficientes } \\
\text { estandarizados }\end{array}$ & $T$ & $p$ \\
\hline & & $B$ & E.T. & Beta & & \\
\hline & $\begin{array}{l}\text { Número de fijaciones } \\
\text { BALÓN-MUÑECA (BM) }\end{array}$ & 28.76 & 10.174 & 0,45 & 2.827 & 0,008 \\
\hline & $\begin{array}{l}\text { Número de fijaciones } \\
\text { CABEZA (CB) }\end{array}$ & -16.071 & 7.093 & $-0,361$ & -2.266 & 0,03 \\
\hline $\begin{array}{l}R^{2}: \\
0,220\end{array}$ & & & & & & \\
\hline
\end{tabular}

En cuanto al modelo de regresión para la variable \%A4, podemos observar como el número medio de fijaciones en la zona $\mathrm{CB}$ afecta negativamente al porcentaje de aciertos (tabla 6).

Tabla 6. Modelos de regresión en función del número de fijaciones. Variable dependiente: \%A4.

\begin{tabular}{|c|c|c|c|c|c|c|}
\hline & & \multicolumn{2}{|c|}{$\begin{array}{l}\text { Coeficientes no } \\
\text { estandarizados }\end{array}$} & \multirow{2}{*}{$\begin{array}{c}\begin{array}{c}\text { Coeficientes } \\
\text { estandarizados }\end{array} \\
\text { Beta } \\
\end{array}$} & \multirow[t]{2}{*}{$T$} & \multirow[t]{2}{*}{$p$} \\
\hline & & $B$ & E.T. & & & \\
\hline & $\begin{array}{l}\text { Número de fijaciones } \\
\text { CABEZA (CB) }\end{array}$ & -21.16 & 7.089 & $-0,499$ & -2.985 & 0,005 \\
\hline $\begin{array}{l}R^{2}: \\
0,263\end{array}$ & & & & & & \\
\hline
\end{tabular}

No se obtiene un modelo adecuado que permita predecir el \%A3, ya que el coeficiente de correlación obtenido es muy bajo. 


\section{DISCUSIÓN}

El objetivo del trabajo es conocer la información visual en la que centra su atención la bloqueadora. Concretamente, la finalidad del análisis es conocer si el número y duración de fijaciones visuales de las participantes influye en el acierto o error en su toma de decisiones.

En cuanto a la influencia de la duración media de las fijaciones sobre las variables de porcentaje de aciertos, encontramos que la duración prolongada de las fijaciones en la zona $\mathrm{HC}$ y en la zona $\mathrm{CB}$ influían negativamente sobre el porcentaje de aciertos, tanto en el total como en zona 3 y 4.

Estas zonas son de las más fijadas por parte de las participantes del estudio, ocupando el segundo lugar la zona CB y el tercero HC. Teniendo en cuenta que la zona CB puede ser considerada como un pivote visual, podemos explicar que esta influencia negativa sobre el porcentaje de aciertos es debida a que esta localización sirve para captar información relevante de varias fuentes de la escena a través de la visión periférica, pero que es la visión fóvea la que permite al deportista procesar con detalle lo que sucede en la escena. En situaciones de 1x1, ya que en la escena no aparecen las jugadoras atacantes, parece necesaria la recogida de información precisa e inmediata, obligando al uso de la visión fóvea la mayor parte del tiempo (Williams y Davids, 1998). Emplear mucho tiempo en la zona CB podría ocasionar una pérdida de información detallada e importante de otras zonas de mayor aporte informativo, por lo que sus decisiones pueden llegar a ser peores. En otros estudios desarrollados con anterioridad, la localización de las fijaciones en la zona de la cabeza del lanzador se relacionaba con un patrón visual característico de los novatos (Goulet, Bard y Fleury, 1989), mientras que los expertos preferían fijar su atención en las zonas del brazo efector (Huys et al., 2009; Bourne, Bennett, Hayes, Smeeton y Williams, 2013), considerándose éste como poseedor de información relevante.

En el caso de la duración de las fijaciones en $\mathrm{HC}$, es conveniente resaltar que esta zona aporta información en el momento medio de la acción de la colocadora, y que, pasado este momento, son otras las localizaciones relevantes (Vila-Maldonado, Sáez-Gallego, Abellán y Contreras, 2015). Por ello, es lógico pensar que la jugadora no puede emplear demasiado tiempo fijando $\mathrm{HC}$ o sus resultados serían peores.

En función del número medio de las fijaciones, podemos observar como el número de fijaciones sobre las zonas BM y CB influían sobre el \%AT. A más fijaciones realizadas sobre la zona $B M$ mejores porcentajes de acierto, lo que sucede de forma inversa en el caso de la zona $\mathrm{CB}$, que cuanto más veces es fijada, peores resultados se obtienen en esta variable. 
En el caso de las fijaciones a zona CB se corrobora lo señalado con anterioridad acerca del carácter de esta localización, que no aporta información por sí sola sino que sirve como pivote visual para extraer información de áreas relevantes cercanas a ella. Por tanto, utilizar en demasiadas ocasiones este recurso de pivote repercute en una peor toma de decisiones, ya que impediría fijar la atención en otras zonas de mayor relevancia informativa.

En el caso de las fijaciones a BM, esta zona ofrece información relevante sobre la muñeca de la colocadora y el balón, sobre todo en las fases finales de la acción en la que ambas localizaciones se unen en el momento del contacto. Con el fin de engañar a la defensa contraria, las colocadoras eliminan la flexo-extensión de las extremidades inferiores y superiores, ayudándose más de las muñecas para impulsar el balón a una zona u otra del campo (Ureña, 2007), de modo que fijar este segmento puede ser de gran ayuda para la toma de decisiones de las bloqueadoras.

\section{CONCLUSIONES}

A modo de conclusión, según el modelo obtenido, pueden darse mejores porcentajes de aciertos con un mayor número de fijaciones a la zona BM y un menor número de fijaciones a la zona $\mathrm{CB}$. En cuanto a la duración, los mejores resultados se dan empleando el menor tiempo posible en las fijaciones hacia CB y HC.

\section{REFERENCIAS BIBLIOGRÁFICAS}

Abernethy, B. (1988). Visual search in sport and ergonomics: its relationship to selective attention and performer expertise. Human Performance, 4, 205235. https://doi.org/10.1207/s15327043hup0104_1

Abernethy, B. (1990). Expertise, visual search and information pick-up in squash. Perception, 19, 63-77. https://doi.org/10.1068/p190063

Abernethy, B., Neal, R. J. y Koning, P. (1994). Visual-Perceptual and cognitive differences between expert, intermediate and novice snooker players. Applied Cognitive Psychology, 8, 185-211. https://doi.org/10.1002/acp.2350080302

Afonso, J., Garganta, J., McRobert, A., Williams, A. M. y Mesquita, I. (2012). The perceptual cognitive processes underpinning skilled performance in volleyball: Evidence from eye-movements and verbal reports of thinking involving an in situ representative task. Journal of Sports Science and Medicine, 11, 339-345.

Bard, C. y Fleury, M. (1976). Analysis of visual search activity during sport problem situations. Journal of Human Movement Studies, 3, 214-222.

Bourne, M., Bennett, S. J., Hayes, S. J., Smeeton, N. J. y Williams, M. (2013). Information underpinning anticipation of goal-directed throwing. Attention, 
Perception and Psychophysics, 75, 1559-1569. https://doi.org/10.3758/s13414-013-0485-2

Caserta, R. J., Young, J. y Janelle, C. M. (2007). Old dogs, new tricks: training the perceptual skills of senior tennis players. Journal of Sport \& Exercise Psychology, 29, 479-497. https://doi.org/10.1123/jsep.29.4.479

Damas, J. S., Moreno, F. J., Reina, R. L. y Del Campo, V. (2004). Presentación de un sistema automatizado para el análisis de la eficacia de los receptores en voleibol. Motricidad, 11, 105-119.

Del Campo, V. L., Reina, R., Sanz, D., Fuentes, J. P. y Moreno, F. J. (2003). Análisis del comportamiento visual y de reacción de tenistas de diferente nivel ante la simulación en laboratorio de la situación de aproximación a la red. Kronos, 4, 29-38.

Goulet, C., Bard, C. y Fleury, M. (1989). Expertise differences in preparing to return a tennis serve: A visual information processing approach. Journal of Sport and Exercise Psychology, 11(4), 382-398. https://doi.org/10.1123/jsep.11.4.382

Hayhoe, M. M. (2004). Advances in relating eye movements and cognition. Infancy, 6(2), 267-274. https://doi.org/10.1207/s15327078in0602_7

Huys, R., Cañal-Bruland, R., Hagemann, N., Beek, P. J., Smeeton, N. J. y Williams, A. M. (2009). Global information pickup underpins anticipation of tennis shot direction. Journal of Motor Behavior, 41, 158-170. https://doi.org/10.3200/JMBR.41.2.158-171

Just, M. A. y Carpenter, P. A. (1976). The role of Eye Fixations research in cognitive psychology. Behavioral research methods and instrumentation, 8, 139-143. https://doi.org/10.3758/BF03201761

Kioumourtzoglou, E., Derri, V., Tzetzis, G. y Therodorakis, Y. (1998). Cognitive, perceptual and motor abilities in skilled basketball performance. Perceptual \& Motor Skills, 86, 771-786. https://doi.org/10.2466/pms.1998.86.3.771

Kioumourtzoglou, E., Michalopoulou, M., Tzetzis, G. y Kourtessis, T. (2000). Ability Profile of the élite volleyball player. Perceptual \& Motor Skills, 90, 757-770. https://doi.org/10.2466/pms.2000.90.3.757

Knudson, D. y Kluka D. A. (1997). The impact of vision and vision training on sport performance. Journal of Physical Education, Recreation and Dance, 68, 1724. https://doi.org/10.1080/07303084.1997.10604922

Liebermann, D. G., Katz, L., Hughes, M. D., Bartlett, R. M., McClements, J. y Franks, I. M. (2002). Advances in the application of information technology to sport performance. Journal of Sports Sciences, 20, 755-769. https://doi.org/10.1080/026404102320675611

Mann, D., Williams, A. M., Ward, P. y Janelle, C. (2007) Perceptual-cognitive expertise in sport: A meta-analysis. Journal of Sport \& Exercise Psychology, 29, 457-478. https://doi.org/10.1123/jsep.29.4.457

Moreno, F. J, Ávila, F. y Damas, J. S. (2001). El papel de la motilidad ocular extrínseca en el deporte. Aplicación en los deportes abiertos. Motricidad, 7 , 75-94. 
Moreno, F. J., Del Campo, V., Reina, R. L., Ávila, F. y Sabido, R. (2003). Las estrategias de búsqueda visual seguidas por los deportistas y su relación con la anticipación en el deporte. Cuadernos de psicología del deporte, 3(1), 7-13.

Palmi, J. (2007). La percepción: enfoque funcional de la visión. Apunts Educación Física y Deportes, 2, 81-85.

Quevedo, L. y Solé, J. (2007). Visión periférica: propuesta de entrenamiento. Apunts Educación Física y Deportes, 2, 75-80.

Reina, R., Moreno, F. J. y Sanz, D. (2007). Visual behaviour and motor responses of novice and experienced wheelchair tennis players relative to the service return. Adapted Physical Activity Quarterly, 24, 254-271. https://doi.org/10.1123/apaq.24.3.254

Roca, A., Ford, P. R., McRobert, A. P. y Williams, A. M. (2013). Perceptual-Cognitive Skills and Their Interaction as a Function of Task Constraints in Soccer. Journal of Sport and Exercise Psychology, 35, 144-155. https://doi.org/10.1123/jsep.35.2.144

Ruiz, L. M. y Sánchez, F. (1997). Rendimiento Deportivo. Claves para la optimización de los aprendizajes. Madrid: Gymnos

Savelsbergh, G., Williams, A. M., Van der Kamp, J. y Ward, P. (2002). Visual Search, anticipation and expertise in soccer goalkeepers. Journal of Sports Sciences, 20, 279-287. https://doi.org/10.1080/026404102317284826

Scheiter, K. y Van Gog, T. (2009). Using Eye Tracking in Applied Research to Study and Stimulate the Processing of Information from Multi-representational Sources. Applied Cognitive Psychology, 23, 1209-1214. https://doi.org/10.1002/acp.1524

Sellinger, A. y Ackerman, J. (1985). El voleibol de potencia. Buenos Aires: Confederación Argentina de Voleibol.

Tenebaum, G. (2003). Expert athletes: an integrated approach to decision making. En J. L. Starkes y K. A. Ericsson (Eds.). Expert performance in Sports, advances in research on sport expertise. (pp. 191-218). Champaing: Human Kinetics.

Ureña, A. (2007). La técnica. Curso Nacional de Entrenadores de Voleibol Nivel III. Cáceres: Real Federación Española de Voleibol.

Vaeyens, R., Lenoir, M., Williams, A. M., Mazyn, L. y Philippaerts, R. M. (2007). The effects of task constraints on visual search behaviour and decision-making skill in youth soccer players. Journal of Sport \& Exercise Psychology, 29, 147-169. https://doi.org/10.1123/jsep.29.2.147

Vaeyens, R., Lenoir, M., Williams, A. M. y Philippaerts, R. M. (2007). Mechanisms underpinning successful decision making in skilled youth soccer players: an analysis of visual search behaviors. Journal of Motor Behavior, 39(5), 395408. https://doi.org/10.3200/JMBR.39.5.395-408

Vickers, J.N. (1988). Knowledge structures of élite-novice gymnasts. Human Movement Science, 7, 47-72. https://doi.org/10.1016/0167-9457(88)900048 
Vickers, J.N. (2007). Perception, cognition and decision training. The quiet eye in action. Champaing: Human Kinetics.

Vila-Maldonado, S., Sáez-Gallego, N. M., Abellán, J. y García-López, L. M. (2014). Análisis de la toma de decisiones en la acción del bloqueo en voleibol; Comparación entre jugadoras de élite y amateur. Revista de Psicología del Deporte, 23(2), 239-246.

Vila - Maldonado, S., Sáez-Gallego, N. M., Abellán, J. y Contreras, O. R. (2015). Patrón visual de jugadoras experimentadas de voleibol durante la acción del bloqueo. Cultura, Ciencia y Deporte, 30(10), 245-254. https://doi.org/10.12800/ccd.v10i30.593

Williams, A. M. y Davids, K. (1995). Declarative knowledge in sport: a by-product of experience or a characteristic of expertise? Journal of Sport and Exercise Psychology, 17, 259-275. https://doi.org/10.1123/jsep.17.3.259

Williams, A. M. y Davids, K. (1998). Visual search strategy, selective attention and expertise in soccer. Research Quaterly for Exercise and Sport, 69(2), 111129. https://doi.org/10.1080/02701367.1998.10607677

Williams, A. M. (2000). Perceptual skill in soccer: implications for talent identification and development. Journal of Sports Sciences, 18, 1-14. https://doi.org/10.1080/02640410050120041 https://doi.org/10.1080/02640410050120113

Williams, A. M., Davids, K., Burwitz, L. y Williams, J. G. (1993). Visual search and sports performance. Australian Journal of Science and Medicine in Sport, 22, 55-65.

Williams, A. M., Singer, R. N. y Frehlich, S. G. (2002). Quiet eye duration, expertise, and task complexity in near and far aiming task. Journal of Motor Behavior, 34(2), 197-207. https://doi.org/10.1080/00222890209601941

Total references / Referencias totales: 40 (100\%).

Journal's own references / Referencias propias de la revista: $0(0 \%)$.

Rev.int.med.cienc.act.fís.deporte - vol. 19 - número 75 - ISSN: 1577-0354 